\title{
Tumour-infiltrating lymphocytes and response to neoadjuvant letrozole in patients with early oestrogen receptor- positive breast cancer: analysis from a nationwide phase II DBCG trial
}

\author{
Signe Korsgaard Skriver ${ }^{1 *}$ (D, Maj-Britt Jensen², Ann Soegaard Knoop ${ }^{1}$, Bent Ejlertsen ${ }^{1,2}$ and
} Anne-Vibeke Laenkholm ${ }^{3}$

\begin{abstract}
Background: The presence of tumour-infiltrating lymphocytes (TILs) is associated with response to neoadjuvant chemotherapy among patients with triple-negative and HER2-positive breast cancer. However, the significance of TILs is less clear in luminal breast cancer. Here, we in postmenopausal patients with primary oestrogen receptorpositive (ER+), HER2 normal, operable breast cancer assessed the importance of inducing TILs during 4 months of letrozole on response in a neoadjuvant phase II study.

Methods: Participants were postmenopausal women with ER+, HER2 normal operable breast cancer assigned to 4 months of neoadjuvant letrozole. Pretreatment core biopsies and surgical specimens were assessed centrally for the percentage of TILs on haematoxylin and eosin-stained slides according to the International Immuno-Oncology Biomarker Working Group on Breast Cancer guidelines. Pathological response was assessed by the Residual Cancer Burden (RCB) index and a modified Miller-Payne grading system and was analysed according to change in TILs.

Results: Tumour specimens were available from 106 of the 112 patients treated per protocol. TIL concentration increased with mean 6.8 percentage point $(p<0.0001)$ during treatment (range -39 to 60$)$. An increase in TILs was significantly associated with pathological response with $\mathrm{OR}=0.71(95 \% \mathrm{Cl} 0.53-0.96 ; p=0.02)$ per $10 \%$ absolute increase for pathological response and correspondingly $\mathrm{OR}=0.56(95 \% \mathrm{Cl} 0.40-0.78 ; p=0.0007)$ for lower $\mathrm{RCB}$ index per 10\% increase.
\end{abstract}

Conclusion: Increasing TILs during letrozole was significantly associated with a poor treatment response. An increase in TILs during endocrine therapy might imply immunogenicity, and these patients could be targetable by immunotherapy.

Trial registration: ClinicalTrials.gov NCT00908531, registered 27 May 2009.

Keywords: Breast neoplasms, Neoadjuvant, Endocrine therapy, Letrozole, TILS

\footnotetext{
* Correspondence: signe.korsgaard.skriver@regionh.dk

'Department of Oncology, Rigshospitalet, Copenhagen University Hospital, section 5703 Rigshospitalet, Blegdamsvej 9, DK-2100 Copenhagen, Denmark Full list of author information is available at the end of the article
}

(c) The Author(s). 2020 Open Access This article is licensed under a Creative Commons Attribution 4.0 International License, which permits use, sharing, adaptation, distribution and reproduction in any medium or format, as long as you give appropriate credit to the original author(s) and the source, provide a link to the Creative Commons licence, and indicate if changes were made. The images or other third party material in this article are included in the article's Creative Commons licence, unless indicated otherwise in a credit line to the material. If material is not included in the article's Creative Commons licence and your intended use is not permitted by statutory regulation or exceeds the permitted use, you will need to obtain permission directly from the copyright holder. To view a copy of this licence, visit http://creativecommons.org/licenses/by/4.0/ The Creative Commons Public Domain Dedication waiver (http://creativecommons.org/publicdomain/zero/1.0/) applies to the data made available in this article, unless otherwise stated in a credit line to the data. 


\section{Background}

Tumour-infiltrating lymphocytes (TILs) have been established as a predictive biomarker for response to neoadjuvant chemotherapy irrespective of molecular subtype [1]. The evidence is most pronounced in triple-negative and HER2-positive breast cancer, and likewise, increased TILs are a strong prognostic factor for improved survival in early triple-negative and HER2-positive breast cancer $[2,3]$. In contrast, the presence of TILs has in oestrogen receptor-positive $(\mathrm{ER}+)$ breast cancer been suggested to be an adverse prognostic factor and associated with poor response to aromatase inhibitors $[1,4,5]$. While the composition and dynamics of TILs are complex, several studies have shown that the predictive information from immune gene expression analyses correlates well with TIL count on haematoxylin and eosin-stained slides $[6,7]$.

Neoadjuvant studies have a major strength that by comparing sequential specimens from patients before and after treatment it is possible to obtain predictive and prognostic information using tumour response, linking biology with clinical response. Response to neoadjuvant treatment can be used to stratify post neoadjuvant treatment. Pathological complete response (pCR) has been the most commonly used endpoint in neoadjuvant trials, but a low pCR rate in ER+ breast cancer makes pCR a less than ideal endpoint in this population. Different pathological scoring systems for residual disease (RD) exist including the Miller-Payne grading system, which estimates a decrease in cellularity during treatment [8], and the Residual Cancer Burden (RCB) index that take tumour dimensions, cellularity of the tumour bed and axillary nodal burden into account $[9,10]$. For neoadjuvant endocrine treatment, the Preoperative Endocrine Therapy Prognostic Index (PEPI) score has been applied in some occasions [11]. The PEPI score is however not routinely used mainly due to the lack of standardisation and validity of Ki67 scoring [12].

In this study, we investigated the dynamics of TILs and relationship to pathological outcome during neoadjuvant endocrine therapy.

\section{Methods}

\section{Study population}

Patients were treated with neoadjuvant letrozole for 4 months prior to curative intended surgery as part of a clinical phase II study conducted by the Danish Breast Cancer Group (DBCG) between 2009 and 2012. The study is registered on clinicaltrials.gov (NCT00908531). The original study design and clinical results have been previously published [13]. Endpoints were clinical and pathological outcome. A total of 119 patients were registered to receive neoadjuvant letrozole. Eligible patients had histological confirmed, invasive, ER+, HER2negative, operable breast cancer. They met the following criteria: tumour size $\geq 1 \mathrm{~cm}, \geq 60$ years at entry, Eastern Cooperative Oncology Group score 0-2 and Charlson comorbidity index $0-2$. Patients with prior cytotoxic treatment including aromatase inhibitors and prior malignant disease were not eligible. Patients were registered in the DBCG database and updated prospectively. Four patients were excluded prior to study initiation, two cases due to HER2 positivity at central testing and two patients withdrew consent. An additional three were excluded from the intention-to-treat population. One hundred six patients had paired tissue samples from biopsy and surgery and were included in the present study (supplementary Fig. 1).

\section{Biomarker analyses}

ER, PGR, HER2 and Ki67 were assessed centrally using international standards [14-16]. ER and Ki67 were recorded as continuous variables. Tumours were considered ER positive when nuclear staining was equal to or higher than $10 \%$. TILs were assessed by the use of the guidelines of the International Immuno-Oncology Biomarker Working Group on Breast Cancer [17]. Pre- and posttreatment stromal TILs were quantified on haematoxylin and eosin-stained slides, from biopsies and surgical specimens respectively, as percentage infiltration of mononuclear cells. TILs were assessed both continuously and categorised into groups: low (0-9\%), intermediate (10-59\%) and high (60-100\%). Changes in TILs were calculated as the difference between pre- and posttreatment TIL counts and categorised as decreased when TILs dropped $\geq 10$ percentage point, as increased when TILs elevated $\geq 10$ percentage point or as no change (less than 10 percentage point decrease or increase).

\section{Endpoint}

Endpoint was to determine the association between changes in TILs during neoadjuvant endocrine treatment and achieving a pathological response. Pathological response was defined as a decrease in tumour cells $\geq 30 \%$ according to a modified Miller-Payne grading scale used by the DBCG [8]. On the modified scale response, grade 1 equals no invasive cells present in the tumour bed, pathological complete response (pCR). Grade 2 more than $90 \%$ loss of tumour cells and grade 3 between 30 and $90 \%$ reduction in tumour cells were considered partial response. Grade 4 is defined as less than $30 \%$ loss of tumour cells and was considered no response. Exploratively pathological response was also assessed on the RCB index scoring RD both continuously and categorically using the guidelines presented by the BIG-NABCG collaboration $[9,10]$. In brief, the RCB index combines the bidimensional diameter of the primary tumour with the percentage of invasive cells in the tumour, corrected for the percentages of in situ carcinoma, and the number 
of positive lymph nodes including the diameter of the biggest lymph node metastasis in a generalised linear model. The higher RCB index, and the corresponding RCB-class, the more extensive residual disease load. For the RCB index calculations in this study, we used the online Residual Cancer Burden Calculator provided by the MD Andersson Cancer Center [18]. As an additional exploratory analysis, we assessed response after the PEPI scoring system. The PEPI score combines pathological tumour size, ER, Ki67 and node status in a score from 0 to 12 where a low PEPI score indicates excellent prognosis.

\section{Statistical methods}

The distribution of TILs in standard clinicopathological subgroups was tested with chi-square or Fisher's exact test. The distribution of changes in TILs did not meet the assumption of normality, and the Wilcoxon signed rank test was used to test for changes in distribution pre- and posttreatment. The association with pathological outcome was tested with univariate logistic regression. Factors were included in univariate models both categorically and continuously to investigate the functional form. Unknowns were included in separate categories. Multivariate analyses including factors that were statistically significant in the univariate analyses were applied to assess the adjusted odds ratios. Factors that were not statistically significant in the multivariate analysis were excluded from the final model. Odds ratio (OR) was estimated with a $95 \%$ confidence interval (CI), using the category with the highest number of patients as the reference group, except for PGR to align it with ER. The exploratory variables RCB and PEPI score were only tested in univariate analysis. Correlation between TILs and other biomarkers was tested with Pearson's correlation. The level of significance was set to $5 \%$. All $p$ values are two-sided. All analyses were performed with SAS enterprise guide version 7.15 (Cary, NC, USA).

\section{Results}

\section{Patient demographics and pretreatment TILs}

Patients' basic characteristics are summarised in Table 1. Median age was 67 years (range 60-87 years). All patients had pretreatment TIL assessment, with a baseline median of $5 \%$, range $0-50 \%$. Apart from Ki67, there were no statistically significant differences between patient with low and intermediate levels of pretreatment TILs in the different clinicopathological subgroups (Table 1).

\section{Pathological response}

Assessment of pathological response according to Miller-Payne was available for 102 patients (96\%). Of them, 58 (57\%) had pathological response including one patient with pCR. Forty-four (43\%) had no response. Assessment of RCB was possible for all 106 patients: range RCB index 0-3.649, and when grouped into RCBclasses: one patient had RCB-class 0 (pCR), six (6\%) had RCB-class I, 90 (83\%) had RCB-class II and 12 (11\%) had RCB-class III. Assessment of the PEPI score was possible for 104 patients, with $45(43 \%)$ patients obtaining a PEPI score of 0 and 59 (57\%) a PEPI score between 1 and 6 (mean score 1.7) (supplementary Table 1).

\section{TIL changes during treatment}

TIL concentration increased overall with a mean of 6.8 $(p<0.0001)$ during treatment (range -39 to 60$)$. Thirty-nine (37\%) patients had an increase in TIL concentration of $10 \%$ or higher (mean $23 \%$, range $10-60 \%$ ), $12(11 \%)$ had a decrease in TIL concentration (mean drop 19\%, range $10-39 \%$ ) and the remaining 55 patients had stable TILs.

When divided into responders and non-responders, the increase in concentration was significant among non-responders with a mean increase of 10.6 $(p<0.0001)$ compared to responders (mean increase 3.2, $p=0.10$ ); these data are presented in Table 2 and Fig. 1.

\section{Variables' relation to outcome}

All clinicopathological factors listed in Table 1 are tested for association to pathological response. Tumour size below $2 \mathrm{~cm}$, a ductal subtype, low posttreatment Ki67 and stable or decreasing TILs were predictive of pathological response in the univariate analysis. When tested in multivariable analyses, only TILs and subtype remained significant for pathological response. In the final model, OR estimates for pathological response were 0.71 (95\% CI $0.53-0.96$; $p=0.02$ ) per $10 \%$ absolute increase in TILs and 0.37 (95\% CI $0.15-0.91 ; p=0.03$ ) for non-ductal subtype as presented in Table 3. For the explorative endpoint, RCB TILs were significantly predictive for response with $\mathrm{OR}=0.56$ (95\% CI 0.40 $0.78 ; p 0.0007)$ per $10 \%$ absolute increase in TILs and posttreatment Ki67 was borderline significant with OR 0.77 (CI $0.60-1.00 ; p=0.05$ ) per $10 \%$ increase in Ki67 index. There was no significant association between TILs and achievement of a PEPI score of 0 : OR 0.85 (CI $0.66-1.10 ; p=0.22$ ) per $10 \%$ increase in TILs.

\section{Correlation between TILs and standard pathological variables}

Ki67 levels paralleled pretreatment TIL levels. The correlation between pretreatment TILs and Ki67 was moderate (Pearson $0.4 ; p=0.0002$ ); however, the association grew stronger posttreatment (Pearson $0.5 ; p<0.0001$ ). TILs were weakly correlated to ER (Pearson $-0.2 ; p=$ 0.02 pretreatment and $-0.3 ; p=0.0006$ posttreatment) 
Table 1 Patient and tumour characteristics of the study population

\begin{tabular}{|c|c|c|c|c|c|c|c|}
\hline \multirow[b]{2}{*}{ Characteristic } & \multicolumn{2}{|c|}{ All patients, $n=106$} & \multicolumn{2}{|c|}{ Patients with low TILs, $n=81$} & \multicolumn{2}{|c|}{ Patients with intermediate TILs, $n=25$} & \multirow[b]{2}{*}{$p^{1}$} \\
\hline & $n$ & $\%(\mathrm{col})$ & $n$ & $\%(\mathrm{col})$ & $n$ & $\%(\mathrm{col})$ & \\
\hline Age (years) & & & & & & & 0.12 \\
\hline $60-69$ & 44 & (41) & 44 & (54) & 18 & $(72)$ & \\
\hline $70-89$ & 62 & (59) & 37 & (46) & 7 & (28) & \\
\hline
\end{tabular}

Tumour size $(\mathrm{mm})^{2}$

$\begin{array}{lllllll}<20 & 46 & (43) & 33 & (41) & 13 & \text { (52) } \\ \geq 20 & 60 & (57) & 48 & (59) & 12 & \text { (48) }\end{array}$

Histological subtype

$\begin{array}{lll}\text { Ductal } & 76 & (72) \\ \text { Lobular } & 12 & (11) \\ \text { Other invasive } & 17 & (16) \\ \text { Unknown } & 1 & (1)\end{array}$

$\begin{array}{ll}56 & (70) \\ 11 & (14) \\ 13 & (16) \\ 1 & (1)\end{array}$

Malignancy grade 4

$\begin{array}{ll}1 & 37 \\ 2 & 45 \\ 3 & 5\end{array}$

$\begin{array}{lll}37 & (43) & 28 \\ 45 & (52) & 35 \\ 5 & (6) & 4\end{array}$

28
35
4

Axillary node status

$\begin{array}{ll}\text { Negative } & 58 \\ \text { Positive } & 47 \\ \text { Unknown } & 1\end{array}$

(55) $\quad 41$

41

$$
40
$$

Oestrogen receptor status (\%)

(19) 13
13

68

\section{(71)}

2

Unknown

Ki67 index (\%)

$\begin{array}{lll}<14 & 72 & (68) \\ \geq 14 & 32 & (30) \\ \text { Unknown } & 2 & (2)\end{array}$

${ }^{4}$ only lobular and ductal tumours graded, $n=88$

TILS, tumour-infiltrating lymphocytes

Table 2 TIL increase during neoadjuvant letrozole in postmenopausal breast cancer patients $(n=106)$

\begin{tabular}{lll}
\hline & Change & $p$ \\
\hline Overall increase, mean (range) & $6.8(-39$ to 60$)$ & $<\mathbf{0 . 0 0 0 1}$ \\
Responders $(n=58)$ & $3.2(-39$ to 35$)$ & 0.10 \\
Non-responders $(n=44)$ & $10.6(-25$ to 60$)$ & $<\mathbf{0 . 0 0 0 1}$ \\
\hline
\end{tabular}

TILs tumour-infiltrating lymphocytes and not significantly correlated to PgR (Pearson - 0.04; $p=0.70$ pretreatment and $-0.2 ; p=0.21$ posttreatment).

\section{Discussion}

We found that an increase in TILs during neoadjuvant letrozole was associated with a poor treatment response, regardless of the pathological assessment method. Furthermore, TILs were positively correlated with Ki67 levels both before and after 4 months of letrozole. A high Ki67 index is indicative for a luminal B subtype, which harbours a greater genomic instability than luminal A [19]. A high number of mutations increase the 


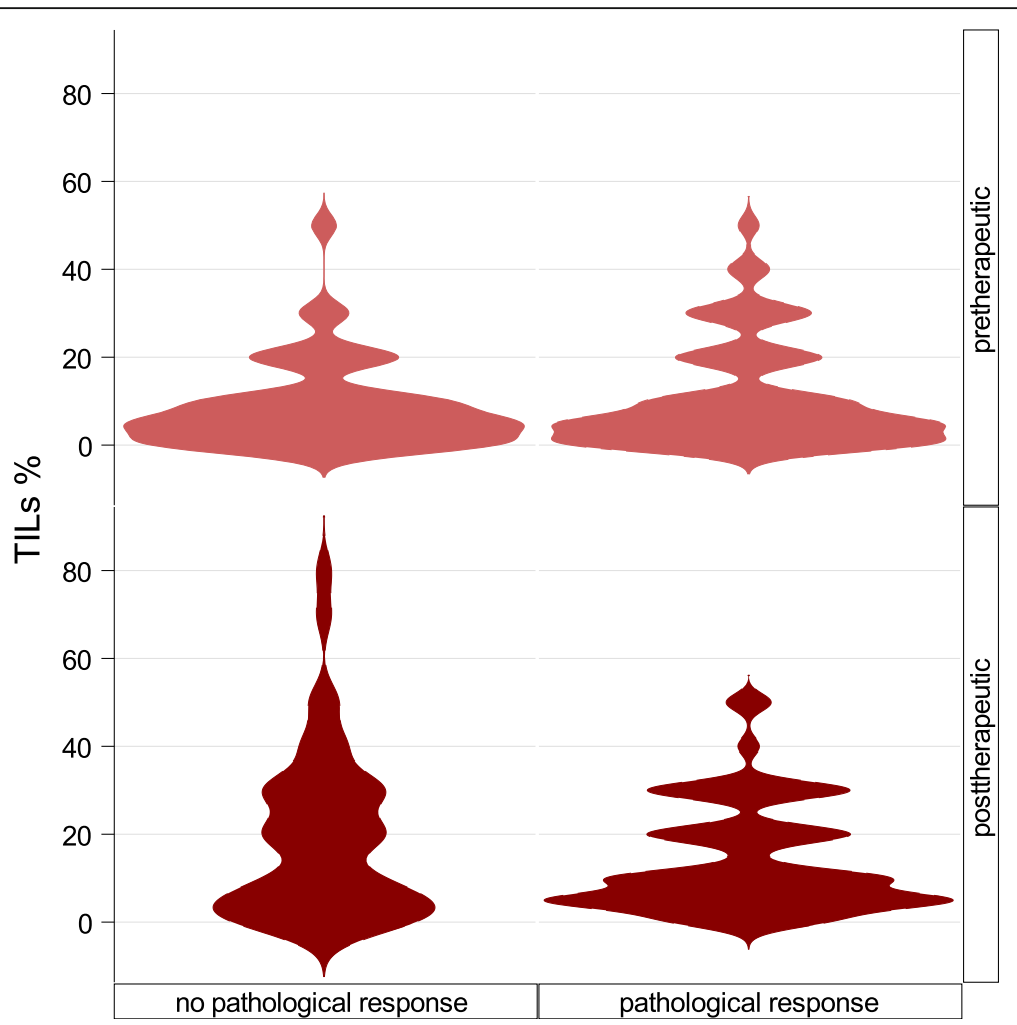

Fig. 1 Bean plot illustrating distributions of TILs before and after neoadjuvant endocrine treatment in patients with no pathological response and in patients with pathological response (complete or partial). Pre- and posttreatment distribution differs significantly in patients with no pathological response $(p \leq 0.0001)$, but not in patients with response $(p=0.1)$

chance that mutated protein sequences will be exposed and recognised as neo-antigens by the immune system, hereby activating the immune system and promoting lymphocytic infiltration of the tumour area. The relationship between Ki67 and TILs in ER+, HER2 normal breast cancer has been examined by others with differing results. Denkert et al. [2] showed that stratifying ER+, HER2 normal breast cancers in high/low Ki67 did not change the overall prognostic effect of TILs, whereas Fujimoto et al. [20] demonstrated that high TILs were associated with favourable DFS in Ki67-high, but not in Ki67-low ER+, HER2 normal breast cancer. As more data will become available, we will gain a deeper understanding of the interaction between the tumour, its microenvironment and the adaptive immune activation system and the implications for prognosis and treatment efficacy.

Our data confirms previous finding by our group and others that tumours of non-ductal histologic subtype predict a poor response to neoadjuvant therapy [13, 21, 22].

Contradictory results regarding TILs were found by Liang et al. in the CARMINA 02 trial [23]. When assessing 83 patients treated with endocrine treatment, they found TILs increased in responders but remained stable in non-responders. As in our study, pretreatment levels of TILs did not predict response. The dissimilarities in our results call for testing in larger studies and alignment of pathological assessment method used. Baseline TILs in our population ranged between 0 and 50\%, which is consistent with the data from Stanton et al. [24] showing that $94 \%$ of ER+ menopausal breast cancer patients had less than $49 \%$ TILs when diagnosed ( $n=$ 2410). Our data supports that of others that ER-positive tumours with higher lymphocytic infiltration have reduced benefit from aromatase inhibitor treatment [4].

The current study has some limitations. Firstly, the small sample size in the study results in a limited power, and secondly, we do not have knowledge of the composition of immune cells beyond TIL count in our population. The strengths of our study include prospectively planned diagnostic and pathological procedures uniformly carried out with central assessment after international recommended guidelines. Patient treatment and follow-up are performed according to DBCG national guidelines [25]. A general limitation for neoadjuvant endocrine studies is the lack of validated methods for response evaluation. The Miller-Payne grading system and the RCB index is both developed and validated in neoadjuvant studies for chemotherapy and does not necessarily translate into the neoadjuvant endocrine setting. The 
Table 3 Univariate and multivariate analyses for pathological response in patients treated with neoadjuvant endocrine letrozole

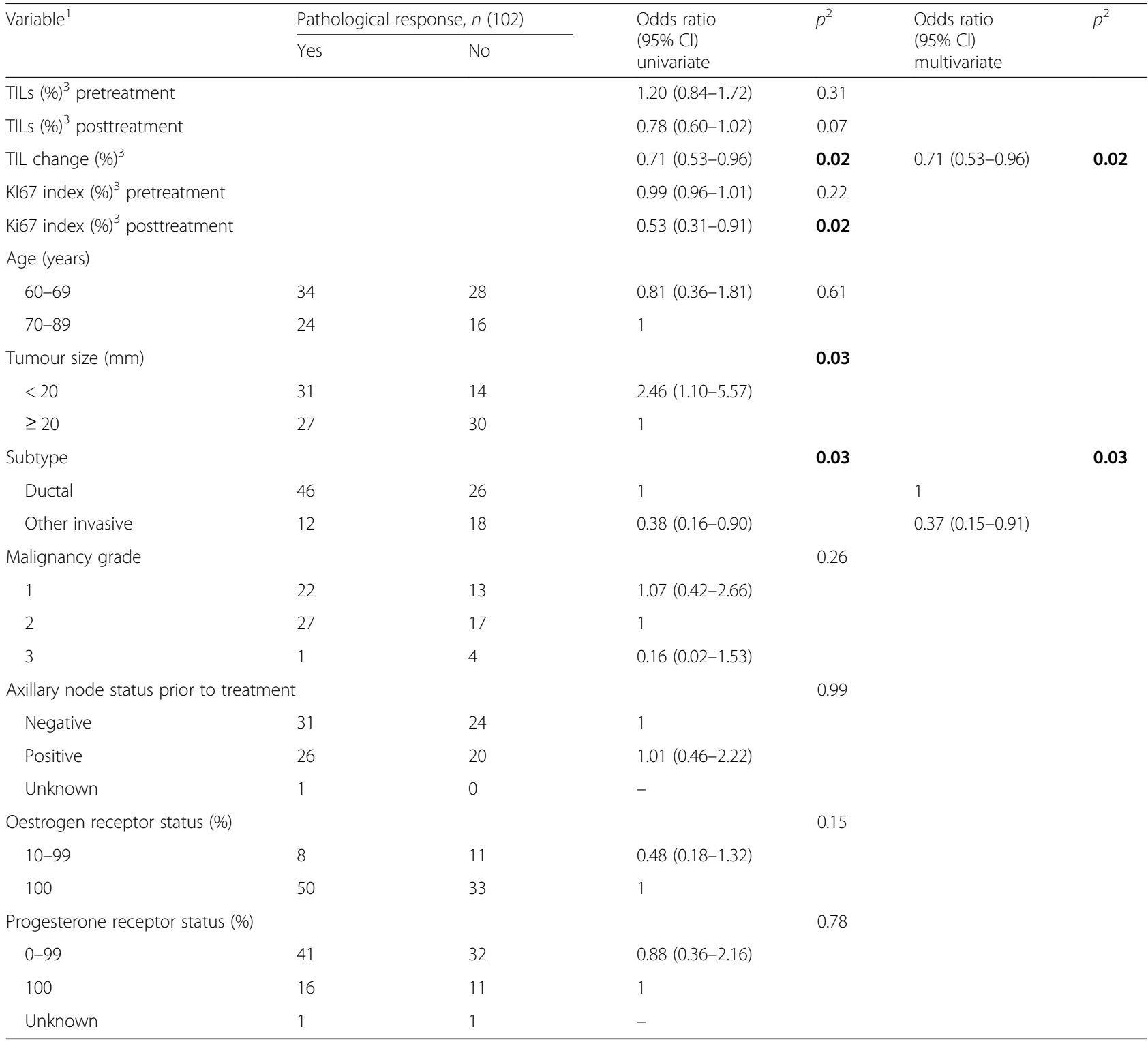

Data reported as $n .{ }^{1}$ Unless otherwise specified, variables are pretreatment values. ${ }^{2}$ Excluding unknowns. ${ }^{3}$ Continuous variable, per $10 \%$ increase TILs, tumour-infiltrating lymphocytes

PEPI score, which has been applied in the neoadjuvant endocrine setting, lacks analytical validity across laboratories rendering it, so far, only feasible for research [12]. The results from this study indicate that RCB might be used for the measurement of neoadjuvant endocrine treatment response since an increase in TILs during treatment was predictive for a poor response, irrespective of the applied response evaluation method.

More knowledge is needed to predict response to endocrine treatment based on the dynamic and composition of TILs, especially more detailed knowledge on the composition of immune cells in luminal breast cancer. Previous studies on TILs in breast cancer identified lymphocyte populations that were mainly comprised of $\mathrm{CD} 8+$ cytotoxic $\mathrm{T}$ cells and $\mathrm{CD} 4+$ regulatory cells together with varying proportion of other helper $\mathrm{T}$ cells, $\mathrm{B}$ cells and NK cells [26]. CD8+ is the key immune cell for tumour cell elimination, working partly through cytotoxic granule release mediated by Granzyme B ( $\mathrm{GrB})$ $[24,27]$. The only known human intracellular inhibitor of $\mathrm{GrB}$ is serine proteinase inhibitor 9 (PI-9). In vitro studies have revealed that elevated levels of oestrogen receptor alpha induce PI-9 [27]. In postmenopausal women, circulating oestrogen is on average $50 \mathrm{pM}$, which is sufficient to induce PI9 [27, 28]. Another study investigating lymphocyte composition changes during 
neoadjuvant endocrine treatment found that oestrogen depletion resulted in a significant increase of the CD8+/ Treg ratio [29].

Breast cancer in general, and especially luminal breast cancer, is considered non-immunogenic, and immunotherapy has yet to have a place in the adjuvant setting for breast cancer patients. TIL count and modulation of immune response could be used to select patients for inclusion in adjuvant immunotherapy trials. Additionally, it is rational to enhance the anti-tumour immune response prior to potential immunotargeted treatment. CDK4/6 inhibitors promote the recruitment of TILs, as a result of several mechanisms including enhanced tumour antigen presentation, reduced proliferation of immunosuppressive regulatory $\mathrm{T}$ cells and a direct stimulatory effect on $\mathrm{T}$ cells $[30,31]$. Although these results are from preclinical studies, the possibilities are promising and possible combinations with targeted and immunotherapy are currently being investigated both in laboratories and in clinical trials.

\section{Conclusions}

Increasing TILs during neoadjuvant endocrine therapy is predictive for a poor pathological response. These patients could be candidates for immunotherapy, but more research on the subject is needed.

\section{Supplementary information}

Supplementary information accompanies this paper at https://doi.org/10. 1186/s13058-020-01285-8.

Additional file 1: Supplementary Fig. 1. Flow diagram of the study population. Supplementary table. Distribution of PEPI score in patients treated with neoadjuvant letrozole between 2009 and 2012.

\section{Abbreviations}

BIG-NABCG: Breast International Group-North American Breast Cancer Group; Cl: Confidence interval; DBCG: Danish Breast Cancer Group; ER+: Oestrogen receptor positive; HER2: Human epidermal growth factor receptor 2; IHC: Immunohistochemistry; OR: Odds ratio; pCR: Pathological complete response; PEPI: Preoperative Endocrine Therapy Prognostic Index; PGR: Progesterone receptor; RCB: Residual Cancer Burden; RD: Residual disease; TILs: Tumour-infiltrating lymphocytes

\section{Acknowledgements}

Not applicable.

\section{Declarations}

A poster based in parts on data from the manuscript has been presented at the San Antonio Breast Cancer Symposium 2019 [32].

\section{Authors' contributions}

$B E, A V L, M J$ and $A S K$ were involved in the conception and design of the original phase II study. All authors acquired the data. SKS and MJ analysed the data. All authors were involved in the interpretation and critical review of the data. SKS drafted the manuscript. All authors read, reviewed and approved the final manuscript.

\section{Funding}

The Danish Cancer Society [grant number R146-A9562]. The founding source had no role in the design or execution of the study and has not reviewed the data or manuscript.

\section{Availability of data and materials}

The data supporting all the figures, tables and supplementary tables in the published article are not publicly available due to institutional restrictions. The dataset can be made available to qualified researchers through application to the Danish Breast Cancer Group. Please contact dbcg. rigshospitalet@regionh.dk.

Ethics approval and consent to participate

All patients provided written informed consent, and the Biomedical Research Ethics of the Danish Capital Region approved the protocol ( $\mathrm{H}-15012740)$.

\section{Consent for publication}

Not applicable.

\section{Competing interests}

SKS: none. MBJ: Nanostring Technologies, Inc. (grant: institutional) and Oncology venture (grant: institutional). ASK: Roche (grant: institutional). Ad Board: Novartis, Astra Zeneca, MDS, Roche, Pfizer, and ELI LILLY DANMARK A/S. BE: Nanostring Technologies, Inc. (grant: institutional), Novartis (grant: institutional), and Roche (grant: institutional). AVL: Nanostring Technologies, Inc. (grant) and Roche (grant).

\section{Author details}

${ }^{1}$ Department of Oncology, Rigshospitalet, Copenhagen University Hospital, section 5703 Rigshospitalet, Blegdamsvej 9, DK-2100 Copenhagen, Denmark. ${ }^{2}$ The Danish Breast Cancer Group, Rigshospitalet, Copenhagen University Hospital, Copenhagen, Denmark. ${ }^{3}$ Department of Surgical Pathology, Zealand University Hospital, Roskilde, Denmark.

Received: 5 February 2020 Accepted: 29 April 2020

Published online: 14 May 2020

References

1. Denkert C, von Minckwitz G, Darb-Esfahani S, Lederer B, Heppner BI, Weber $\mathrm{KE}$, et al. Tumour-infiltrating lymphocytes and prognosis in different subtypes of breast cancer: a pooled analysis of 3771 patients treated with neoadjuvant therapy. Lancet Oncol. 2018;19(1):40-50.

2. Loi S, Drubay D, Adams S, Pruneri G, Francis PA, Lacroix-Triki M, et al. Tumor-infiltrating lymphocytes and prognosis: a pooled individual patient analysis of early-stage triple-negative breast cancers. J Clin Oncol. 2019; 37(7):559-69.

3. Salgado R, Denkert C, Campbell C, Savas P, Nuciforo P, Aura C, et al. Tumorinfiltrating lymphocytes and associations with pathological complete response and event-free survival in HER2-positive early-stage breast cancer treated with lapatinib and trastuzumab. JAMA Oncol. 2015;1(4):448-54.

4. Dunbier AK, Ghazoui Z, Anderson H, Salter J, Nerurkar A, Osin P, et al. Molecular profiling of aromatase inhibitor-treated postmenopausal breast tumors identifies immune-related correlates of resistance. Clin Cancer Res Off J Am Assoc Cancer Res. 2013;19(10):2775-86

5. Solinas C, Carbognin L, De Silva P, Criscitiello C, Lambertini M. Tumorinfiltrating lymphocytes in breast cancer according to tumor subtype: current state of the art. Breast. 2017;35:142-50.

6. Denkert C, Loibl S, Noske A, Roller M, Müller BM, Komor M, et al. Tumorassociated lymphocytes as an independent predictor of response to neoadjuvant chemotherapy in breast cancer. J Clin Oncol. 2009;28(1):105-13.

7. Denkert C, von Minckwitz G, Brase JC, Sinn BV, Gade S, Kronenwett R, et al. Tumor-infiltrating lymphocytes and response to neoadjuvant chemotherapy with or without carboplatin in human epidermal growth factor receptor 2positive and triple-negative primary breast cancers. J Clin Oncol. 2014;33(9): 983-91.

8. Ogston KN, Miller ID, Payne S, Hutcheon AW, Sarkar TK, Smith I, et al. A new histological grading system to assess response of breast cancers to primary chemotherapy: prognostic significance and survival. Breast Edinb Scotl. 2003;12(5):320-7.

9. Symmans WF, Peintinger F, Hatzis $C$, Rajan $R$, Kuerer $H$, Valero V, et al. Measurement of residual breast cancer burden to predict survival after 
neoadjuvant chemotherapy. J Clin Oncol Off J Am Soc Clin Oncol. 2007; 25(28):4414-22.

10. Bossuyt V, Provenzano E, Symmans WF, Boughey JC, Coles C, Curigliano G, et al. Recommendations for standardized pathological characterization of residual disease for neoadjuvant clinical trials of breast cancer by the BIGNABCG collaboration. Ann Oncol. 2015;26(7):1280-91.

11. Ellis MJ, Tao Y, Luo J, A'Hern R, Evans DB, Bhatnagar AS, et al. Outcome prediction for estrogen receptor-positive breast cancer based on postneoadjuvant endocrine therapy tumor characteristics. JNCI J Natl Cancer Inst. 2008;100(19):1380-8.

12. Polley M-YC, Leung SCY, MCShane LM, Gao D, Hugh JC, Mastropasqua MG et al. An international Ki67 reproducibility study. JNCl J Natl Cancer Inst. 2013;105(24):1897-906.

13. Skriver SK, Laenkholm A-V, Rasmussen BB, Handler J, Grundtmann B, Tvedskov TF, et al. Neoadjuvant letrozole for postmenopausal estrogen receptor-positive, HER2-negative breast cancer patients, a study from the Danish Breast Cancer Cooperative Group (DBCG). Acta Oncol. 2018;57(1):31-7.

14. Hammond MEH, Hayes DF, Dowsett M, Allred DC, Hagerty KL, Badve S, et al. American Society of Clinical Oncology/College of American Pathologists guideline recommendations for immunohistochemical testing of estrogen and progesterone receptors in breast cancer. J Clin Oncol. 2010;28(16): 2784-95.

15. Wolff AC, Hammond MEH, Hicks DG, Dowsett M, McShane LM, Allison KH, et al. Recommendations for human epidermal growth factor receptor 2 testing in breast cancer: American Society of Clinical Oncology/College of American Pathologists clinical practice guideline update. J Clin Oncol Off J Am Soc Clin Oncol. 2013;31(31):3997-4013.

16. Dowsett M, Nielsen TO, A'Hern R, Bartlett J, Coombes RC, Cuzick J, et al. Assessment of Ki67 in breast cancer: recommendations from the International Ki67 in Breast Cancer Working Group. JNCl J Natl Cancer Inst. 2011;103(22):1656-64.

17. Dieci MV, Radosevic-Robin N, Fineberg S, van den Eynden G, Ternes N Penault-Llorca F, et al. Update on tumor-infiltrating lymphocytes (TILs) in breast cancer, including recommendations to assess TILs in residual disease after neoadjuvant therapy and in carcinoma in situ: a report of the International Immuno-Oncology Biomarker Working Group on Breast Cancer. Semin Cancer Biol. 2018;52:16-25.

18. Residual Cancer Burden Calculator [Internet]. MD Anderson Cancer Center. [cited 2019 Oct 7]. Available from: http://www3.mdanderson.org/app/ medcalc/index.cfm?pagename=jsconvert3

19. Creighton CJ. The molecular profile of luminal B breast cancer. Biol Targets Ther. 2012;6:289-97.

20. Fujimoto $Y$, Watanabe $T$, Hida Al, Higuchi T, Miyagawa Y, Ozawa $H$, et al. Prognostic significance of tumor-infiltrating lymphocytes may differ depending on Ki67 expression levels in estrogen receptor-positive/HER2negative operated breast cancers. Breast Cancer. 2019;26(6):738-47.

21. Loibl S, Volz C, Mau C, Blohmer J-U, Costa SD, Eidtmann H, et al. Response and prognosis after neoadjuvant chemotherapy in 1,051 patients with infiltrating lobular breast carcinoma. Breast Cancer Res Treat. 2014;144(1): 153-62.

22. Delpech Y, Coutant C, Hsu L, Barranger E, Iwamoto T, Barcenas CH, et al. Clinical benefit from neoadjuvant chemotherapy in oestrogen receptorpositive invasive ductal and lobular carcinomas. Br J Cancer. 2013;108(2): 285-91.

23. Liang X, Briaux A, Becette V, Benoist C, Boulai A, Chemlali W, et al. Molecular profiling of hormone receptor-positive, HER2-negative breast cancers from patients treated with neoadjuvant endocrine therapy in the CARMINA 02 trial (UCBG-0609). J Hematol OncolJ Hematol Oncol. 2018:11(1):124.

24. Stanton SE, Adams S, Disis ML. Variation in the incidence and magnitude of tumor-infiltrating lymphocytes in breast cancer subtypes: a systematic review. JAMA Oncol. 2016;2(10):1354-60.

25. Jensen M-B, Laenkholm A-V, Offersen BV, Christiansen P, Kroman N, Mouridsen HT, et al. The clinical database and implementation of treatment guidelines by the Danish Breast Cancer Cooperative Group in 2007-2016. Acta Oncol Stockh Swed. 2018;57(1):13-8.

26. Savas P, Salgado R, Denkert C, Sotiriou C, Darcy PK, Smyth MJ, et al. Clinical relevance of host immunity in breast cancer: from TILs to the clinic. Nat Rev Clin Oncol. 2016:13(4):228-41.

27. Jiang X, Ellison SJ, Alarid ET, Shapiro DJ. Interplay between the levels of estrogen and estrogen receptor controls the level of the granzyme inhibitor, proteinase inhibitor 9 and susceptibility to immune surveillance by natural killer cells. Oncogene. 2007;26(28):4106-14.

28. Lønning PE, Geisler J. Evaluation of plasma and tissue estrogen suppression with third-generation aromatase inhibitors: of relevance to clinical understanding? J Steroid Biochem Mol Biol. 2010;118(4-5):288-93.

29. Chan MS, Wang L, Felizola SJ, Ueno T, Toi M, Loo W, et al. Changes of tumor infiltrating lymphocyte subtypes before and after neoadjuvant endocrine therapy in estrogen receptor-positive breast cancer patients - an immunohistochemical study of cd8+ and foxp3+ using double immunostaining with correlation to the pathobiological response of the patients. Int J Biol Markers. 2012;27(4):295-304

30. Pernas S, Tolaney SM, Winer EP, Goel S. CDK4/6 inhibition in breast cancer: current practice and future directions. Ther Adv Med Oncol. 2018;10: 1758835918786451

31. Goel S, DeCristo MJ, Watt AC, BrinJones H, Sceneay J, Li BB, et al. CDK4/6 inhibition triggers anti-tumour immunity. Nature. 2017;548(7668):471-5.

32. Skriver SK, Jensen M-B, Knoop AS, Ejlertsen B, Laenkholm A-V. P4-10-18. Changes in tumour infiltrating lymphocytes during neoadjuvant endocrine therapy and possible clinical implications for guiding therapy [Internet]. Poster presented at: San Antonio Breast Cancer Symposium; 2019; San Antonio, TX. [cited 2020 Jan 29]. Available from: https://www. abstractsonline.com/pp8/\#!/7946/presentation/1240.

\section{Publisher's Note}

Springer Nature remains neutral with regard to jurisdictional claims in published maps and institutional affiliations.
Ready to submit your research? Choose BMC and benefit from:

- fast, convenient online submission

- thorough peer review by experienced researchers in your field

- rapid publication on acceptance

- support for research data, including large and complex data types

- gold Open Access which fosters wider collaboration and increased citations

- maximum visibility for your research: over $100 \mathrm{M}$ website views per year

At $\mathrm{BMC}$, research is always in progress.

Learn more biomedcentral.com/submissions 